Methods Between 2009 to 2012 we analysed probes of 11250 individuals (patients and controls) processing the ProbeTecET ${ }^{\circledR}$ test (BD, USA). Of these 407 showed a positive result and were treated according to current guidelines. 33 patients tested negative, however, reported a persistence of discomfort such as burning sensations in the urethra, urethral discharge and occasionally conjunctivitis. These patients were additionally tested with the GenoQuick ${ }^{\circledR}$ CT (HAIN Lifescience, Germany), which specifically and simultaneously detects both, the MOMP-gene and the cryptic plasmide. Material was taken from urethral, cervical, rectal, pharyngeal, conjunctival smears and from the Douglas-space.

Results All 33 patients tested positive when processing the GenoQuick ${ }^{\circledR}$ CT. Thus $7.5 \%$ of infected patients were only identified processing an additional detection set.

Conclusion In our centre $7.5 \%$ of ct infected patients were tested "false negative" when only the cryptic plasmide was analysed. These 33 patients were identified processing a more sensitive test system and subsequently were treated.

\section{P3.023* GEOGRAPHICAL CLUSTERING OF REPEAT POSITIVE TESTS WITH CHLAMYDIA TRACHOMATIS AMONG YOUNG PEOPLE (16-29 YEARS); IDENTIFICATION OF A HIDDEN KEY CHLAMYDIA POPULATION}

doi:10.1136/sextrans-2013-051184.0483

A P A Vermeiren MSc, ${ }^{1,2,3} \mathrm{MD}$ C J P A Hoebe PhD, ${ }^{1,3} \mathrm{G}$ A F S van Liere MSc, ${ }^{4} \mathrm{~B}$ Kauhl MSc, ${ }^{4}$ A Ziemann PhD, ${ }^{4} \mathrm{~T}$ Krafft PhD, ${ }^{1,2,3} \mathrm{~N}$ H T M Dukers-Muijrers PhD. 'Department of Sexual Health, Infectious Diseases and Environmental Health, Public Health Service South Limburg, Geleen, The Netherlands, '2Department of Medical Mircobiology, Maastricht University Medical Centre, Maastricht, The Netherlands, ${ }^{3}$ CAPHRI School of Public Health and Primary Care, Maastricht University Medical Centre, Maastricht, The Netherlands, ${ }^{4}$ Department of International Health, CAPHRI School of Public Health and Primary Care, Faculty of Health, Medicine and Life Sciences, Maastricht, The Netherlands

Background Young patients with repeat infections of Chlamydia trachomatis $(\mathrm{Ct})$ are a key population for prevention as they indicate ongoing risk for spread and complications in women. We estimated the hidden key population, i.e. missed repeat testers and repeat positives, to effectively focus screening strategies.

Methods Data covered all youngsters $(16-29$ years, $n=42,894)$ in Eastern South-Limburg, the Netherlands (2006-2010) including all their genital Ct tests by any care provider. Using logistic regression, determinants (age, sex, socio-economic status (SES)) for not having a repeat test (in positives) and for having a positive repeat test (in repeated testers) were evaluated. Using Geographic-InformationSystems and spatial statistics (SaTScan purely spatial Poisson model, Bivariate Local Moran`s I), spatial clusters and correlations with SES of repeat (positive) tests were evaluated.

Results Overall 10,044 (23.4\%) youngsters were tested of whom $944(9.4 \%)$ were positive. Of positives, $423(44.8 \%)$ had no repeat test (more often older, ORperyear 0.96 95\% CI 0.92-1.00, and male, OR2.2695\% CI 1.69-3.02). Of repeat testers, 111(21.3\%) were repeat positive. Spatial clusters were found in four municipalities(3 low SES) and low SES correlated with repeat positive tests. We estimate that 230 repeat positives ( $0.5 \%$ of total youngsters) are missed in care. These include 90 (21.3\% of 423$)$ who were lost in care for repeat testing follow-up and 140 repeat positives in the 32,850 youngsters who were never tested before (assuming 2.0\% positivity and $21.3 \%$ repeat positivity). Overall, an estimated $67.4 \%(230 /(230+111))$ of all repeat positive patients is thereby missed in current care.

Conclusion Two-thirds of repeat positive patients are hidden to current care, some $(-40 \%)$ because they missed a repeat test and others $(-60 \%)$ because they are never tested. As they comprise a central but small part of the total young population, control strategies targeting this key population should be highly acuminated.
Geo-spatial analysis, which pointed to low SES high prevalence areas informs more effective Ct control.

\section{P3.024 COMPARISON OF CHLAMYDIA TRACHOMATIS ANTIBODIES IN VAGINAL MUCOSA AND SERUM IN WOMEN A FERTILITY CLINIC AND AN STI-CLINIC}

doi:10.1136/sextrans-2013-051184.0484

II V F van den Broek, ${ }^{2} \mathrm{~J}$ A Land, 1,3,4 J E A M van Bergen, 5,6S A Morré, 1,7 M A B van der Sande. 'Epidemiology \& Surveillance Unit, Centre for Infectious Disease Control, National Institute of Public Health and the Environment, Bilthoven, The Netherlands; ${ }^{2}$ Department of Obstetrics and Gynaecology, University Medical Centre, Groningen, The Netherlands; ${ }^{3}$ STI AIDS The Netherlands, Amsterdam, The Netherlands; ${ }^{4}$ Department of General Practice, University of Amsterdam Medical Centre, Amsterdam, The Netherlands; ${ }^{5}$ Laboratory of Immunogenetics, Medical Microbiology and Infection Control, VU University Medical centre, Amsterdam, The Netherlands; 6 Institute of Public Health Genomics, Department of Genetics and Cell Biology, Research Institutes CAPHRI and GROW, University of Maastricht, Maastricht, The Netherlands; 7Julius Centre, University Medical Centre, Utrecht, The Netherlands

Background The common asymptomatic nature of Chlamydia infections and consequential PIDs plus the delayed appearance of any damaging effect thereof on the reproductive tract hamper timely interventions for individuals prone to complications. In infertile women, Chlamydia antibodies in serum relate to tubal pathology and lower conception rates. The current 'proof of principle study' aimed to assess whether Chlamydia antibodies are detectable in easier, non-invasive vaginal mucosa samples, and if these could predict the risk for complications.

Patients and Method We compared outcomes of Chlamydia antibody tests in serum and vaginal swabs in two groups: (a) 77 women attending a fertility clinic, of whom 25 tested positive for anti-chlamydia IgG in serum and (b) 107 women visiting an STI centre, including 30 Chlamydia PCR-positive subjects. The presence of IgG/IgA antibodies was compared (Kappa-test) and determinants investigated (regression)

Results In women in the STI clinic, active Chlamydia infections were linked to both IgG and IgA antibodies in serum $(p<0.001)$ and IgA in vaginal mucosa $(p<0.001)$, but not IgG in mucosa; mucosaIgA correlated with IgG in serum $(p=0.001)$. In women in the fertility clinic, IgG in vaginal mucosal material had a stronger correlation with IgG in serum $(p=0.02)$ than IgA in mucosa $(p=0.06)$. Women with tubal pathology or Chlamydia history more commonly had IgG in serum and IgA in vaginal mucosa (both $p<0.001$ ), whereas this link was weaker for mucosa-IgG $(p=0.03)$; for tubal pathology alone mucosa-IgA had a higher Kappa than serum-IgG (0.41 versus 0.36 ).

Discussion Chlamydia IgG/IgA are detectable in vaginal mucosal material. IgG antibodies in serum had stronger associations with current or past Chlamydia infections. However, IgA antibodies in vaginal mucosa also showed associations with (past) infection and complications. IgA presence in vaginal mucosa might indicate an on-going hidden Chlamydia infection in the upper genital tract, and warrants further epidemiological studies.

\section{P3.025 INTERNET TESTING FOR CHLAMYDIA TRACHOMATIS IN FRANCE IN 2012}

doi:10.1136/sextrans-2013-051184.0485

${ }^{1,2} \mathbf{B}$ de Barbeyrac, ${ }^{3} \mathrm{D}$ Rahib, ${ }^{1} \mathrm{~S}$ de Diego, ${ }^{1,2} \mathrm{C}$ Le Roy, ${ }^{1,2} \mathrm{C}$ Bébéar, ${ }^{3} \mathrm{~N}$ Lydie. ${ }^{1}$ University of Bordeaux, Bordeaux, France, '2Institut National de la Recherche Agronomique, Bordeaux, France, ${ }^{3}$ Institut National de Prévention et d'Education pour la Santé, Saint Denis, France

Chlamydia trachomatis (CT) infection spreads in Europe and in USA, and tendencies analysis shows an increase in the epidemic since last 10 years. In France, in 2006, a national survey carried out by phone and using a home-based sampling showed that CT 
prevalence was the highest in young people, with $3.6 \%$ for women and $2.4 \%$ in men aged $18-24$ yo. To face this problem, several countries have developed new strategies, mixing newer technologies and home-based self-sampling test. Inspired by evaluation of those dispositives, the French National Institute of Health Prevention and Education (INPES) decided to experiment the proposition of a free home-based self-sampling to screen this infection via internet in young people 18-24 yo. This study, named Chlamyweb, aims to compare CT screening rate from this intervention with traditional information system and screening centre. Study design was a random control trial, with a 1:2 randomization. Recruitment took place on an Internet information website on sexually transmitted infections, and support by web campaign from September 3 to October 14 2012. Home-based kits were composed by uriswab 3 sponges for men and dry vaginal swabs for women (Copan diagnostics). All samples were analysed by using the fully automated cobas 4800 (Roche diagnostics). Self-sampling was proposed to 5531 people. Out of them, $47.3 \%$ accepted, with a higher rate in women (53.0\%). A total of 1616 kits provided [1002 from women (63.8\%) and 614 from men (58.8\%)] was return to the French National Reference Center for chlamydial infections. The global prevalence was $6.8 \%$ (8.3\% in women, $4.4 \%$ in men). Sexual behaviour and sociodemographic patient's characteristics were collected and their analysis is under investigation. These preliminary results show that Internet testing reaches a population with a high prevalence of CT infection and appears to be acceptable to young people.

\section{P3.026 LOW PREVALENCE OF CHLAMYDIA TRACHOMATIS INFECTION: FACT OR MISSED DIAGNOSIS?}

doi:10.1136/sextrans-2013-051184.0486

T Bharara, D Rawat, P Bhalla, V K Garg, K Sardana, V Patwardhan. Maulana Azad Medical College, New Delhi, India

Background Chlamydia trachomatis is reported to be the most common sexually transmitted infection (STI) in developed countries; this data depends on the sensitivity and specificity of the diagnostic test employed. Even when Nucleic acid amplification tests (NAATs) are used to detect C.trachomatis infection, the occurrence of variant strains which lack the cryptic plasmid or possess deletion mutations may be responsible for negative test results, as was detected in Sweden recently. The present study was undertaken to establish the true prevalence of C.trachomatis infection among males with urethritis using not only a well tested NAAT but also Direct Fluorescent Antibody test (DFA).

Methods A total of 34 male patients reporting to the STI OPD of the Lok Nayak Hospital with urethritis were included in this study. Gram staining and culture was done for detection of Neisseria gonorrhoeae and DFA and real-time PCR using COBAS ${ }^{\circledR}$ TaqMan $^{\circledR}$ CT Test, v2.0 to detect the presence of C.trachomatis.

Results Among the 34 males, $55.9 \%$ cases were positive for N.gonorrhoeae by culture. DFA for C.trachomatis was positive in $17.6 \%$ cases, 5 out of 6 DFA positive cases were also positive for N.gonorrhoeae. PCR for C.trachomatis has so far been done in 19 cases, including 4 of the DFA positive cases, and none of them yielded a positive result. Neither C.trachomatis nor N.gonorrhoeae was found in $41.1 \%$ cases.

Conclusion C.trachomatis was detected only in $17.6 \%$ men with urethritis and that too only by DFA and none by PCR. This could be either an actual low prevalence of C.trachomatis or it may be due to the occurrence of new variant strains not detected by the molecular diagnostic test used by us. Sequencing of C.trachomatis isolated from patient specimens will be required to detect mutants that could be responsible for underdiagnosis, when relying only on NAATs.

\section{P3.027 PREVALENCE AND DETERMINANTS OF CHLAMYDIA TRACHOMATIS INFECTION AMONG SEXUALLY ACTIVE WOMEN IN TURIN - ITALY}

doi:10.1136/sextrans-2013-051184.0487

D De Maria, G De Intinis, M Peretto, V Buselli, M A Latino. Sant'Anna Hospital, Turin Italy

Background According to the WHO, Chlamydia trachomatis $(\mathrm{Ct})$ is the most common sexually transmitted bacterial agent worldwide. The infection is most common among young women less than 25 years old; the symptoms are often mild or inexistent.

The aim of this study was to assess the prevalence of Chlamydia trachomatis in women in Turin and to identify risk factors associated with this STI.

Methods This study was performed between January 2007 and December 2011, among sexually active women aged 14 to 65 years (mean 33 years) attending STI Centre of Sant'Anna Hospital in Turin, Italy. Every woman was submitted to a questionnaire aimed at collecting socio-demographic, behavioural and clinical information. Cervical specimens were tested with a real time qualitative PCR (Nanogen).

Chi square test and EpiInfo were used to identify associations between potential risk factors and $\mathrm{Ct}$ infection.

Results A total of 14071 women were included in the study. The overall prevalence of $\mathrm{Ct}$ was $2.8 \%$.

The prevalence rate in the symptomatic and asymptomatic patients was $3.5 \%$ versus $2.2 \%$; high prevalence rates were observed among women aged $14-24$ years $(9.9 \%$; $p<0.001)$, reporting more than one partner in the previous six months $(13.3 \%$; $<<0.001)$, among foreign women compared to Italian women (3.7\% vs $2.6 \%$; $\mathrm{p}<0.001)$. With regard to numbers of sexual partners lifetime, the prevalence rate for patients who had between 1 and 3 partners was $1.9 \%$ while in women with more than 3 partners was $4.1 \%$ $(p<0.001)$. In multivariate analysis, Ct was significantly associated with young age, more than 1 partner in the previous 6 months and use of barrier contraceptives.

Conclusion The value and risk factors highlighted can be compared with other studies. The high number of asymptomatic cases confirms the need to consider selective screening in order to reduce the spread of this STI and other secondary complications.

\section{P3.028 POSITIVITY RATE OF CHLAMYDIA TRACHOMATIS AND STATUS QUO OF OPPORTUNISTIC SCREENING IN GERMANY}

doi:10.1136/sextrans-2013-051184.0488

S Dudareva-Vizule, A Sailer, 0 Hamouda, V Bremer. Robert Koch Institute, Berlin, Germany

Background Chlamydia trachomatis (CT) infections are not reportable in Germany and limited data on CT-prevalence among women is available. In 2008 an opportunistic CT screening programme for women $<25$ years (OCS) was introduced. We assessed positivity rate of CT-infection and evaluated the OCS in order to develop recommendations to improve OCS.

Methods In September 2010 voluntary laboratory-based sentinel surveillance system was implemented and is collecting retrospective (from 2008) and prospective data on performed CT-tests together with results and information on age and test reason (TR). We calculated positivity rates (PR) by age and TR and compared them with $\mathrm{Chi}^{2}$ test. We described data by time and estimated the proportion of the target population reached by OCS.

Results As of 27.11.2012 data from 14 laboratories were available for analysis. These data cover approximately $25 \%$ of all CT-tests performed in Germany. Overall $90.6 \%(643,332)$ of all tests $(710,021)$ were among women. Among those $19.1 \%(122,650)$ 